Check for updates

Cite this: RSC Adv., 2019, 9, 30868

Received 24th August 2019

Accepted 9th September 2019

DOI: 10.1039/c9ra06679a

rsc.li/rsc-advances

\section{A high-throughput metabolomics strategy for discovering the influence of differential metabolites and metabolic pathways of huaxian capsules on sepsis-associated Qi deficiency and blood stasis syndrome $\uparrow$}

\begin{abstract}
Qun Liang, (D) *a Han Liu, ${ }^{b}$ Xiu-li Li, ${ }^{a}$ Pei-yang Sun, ${ }^{a}$ Yang Yang ${ }^{a}$ and Chunpeng Du
High-throughput metabolomics can be used to investigate the therapeutic targets and metabolic mechanisms of traditional Chinese medicine (TCM) formulae, which have multiple targets in disease therapy, but it is a great challenge to explore their mechanism of action. Huaxian capsule (HXC) is a classical formula in TCM that has therapeutic effects on a sepsis-associated Qi deficiency and blood stasis syndrome (SQBS). However, its targets and metabolic mechanisms need more investigation. To investigate the therapeutic effects of HXC in the treatment of SQBS and elucidate the potential mechanism, we used a high-throughput metabolomics strategy based on the ultraperformance liquid chromatography/mass spectrometry combined with chemometrics to analyze and identify differential metabolites and pathways. The pathological examination of organs and biochemical indices was also performed to verify the successful establishment of the rat model and protective effects of HXC. Pathological symptoms and biochemical indicators of SQBS rats were reversed by the HXC treatment. A total of 24 potential biomarkers were identified to indicate the difference between the control and model groups; they were closely associated with ten metabolic pathways and regulated by the HXC administration. From the pathway analysis, we further understood the protective activity of HXC against SQBS, which affected amino acid metabolism, molecular transport, small molecule biochemistry and cell signaling as well as vitamin and mineral metabolism. In conclusion, HXC protects against SQBS by modulating the metabolic biomarkers and functional pathways.
\end{abstract}

\section{Introduction}

Sepsis, the main reason for high morbidity and mortality in surgical illness, is characterized by systemic inflammatory responses to infection. ${ }^{1,2}$ It is a common complication of most clinical conditions, such as severe burns, traumas, and surgeries, and is likely to develop into a multiple organ dysfunction, blood coagulation and even death, resulting from the release of various inflammatory mediators. ${ }^{3,4}$ In recent years, sepsis morbidity all over the world has increased gradually by more than $25 \%$. By far, the etiology and pathogenesis of sepsis are still unclear and most studies report that it may be closely related to infection factors, inflammatory response,

${ }^{a}$ ICU Center, First Affiliated Hospital, School of Pharmacy, Heilongjiang University of Chinese Medicine, Heping Road 24, Xiangfang District, Harbin 150040, China. E-mail: liangqun1@sina.com; qunliang1970@163.com; Fax: +86-451-86053141; Tel: +86451-86053141

${ }^{b}$ Simon Fraser University (SFU), Burnaby, British Columbia, Canada

$\dagger$ Electronic supplementary information (ESI) available. See DOI: 10.1039/c9ra06679a immune processes, coagulation changes and multiple organ functions. ${ }^{5}$ Due to the fact that sepsis is harmful to human health, there are many treatments in clinical practice, such as antibiotic, antithrombin, anticytokine and statin therapies. ${ }^{6}$ However, none of these therapies have been successful because of serious side effects or low efficacy. Therefore, there is an urgent need to develop methods to treat sepsis.

Ever since the 2015 Nobel Prize in Physiology or Medicine was awarded for the first time to a Chinese woman scientist, named Youyou Tu, traditional Chinese medicine (TCM) has become the focus of attention in the global research community. ${ }^{7}$ Either primary or secondary metabolites derived from metabolic and biosynthetic enzymes in herbs with diverse, intricate and distinct characteristics exhibit various pharmacological activities. ${ }^{8}$ They have the potential to recover the harmony disturbed by sepsis through multi-target effects of these ingredients. ${ }^{9}$ It was reported that a huaxian capsule (HXC), a traditional heat-clearing formula, has been applied in the treatment of sepsis-associated Qi deficiency and blood stasis syndrome (SQBS). ${ }^{\mathbf{1 0}, 11}$ The protective efficacy of HCX on sepsis was evaluated using limited biochemical 
parameters in most instances, which is inadequate to elaborate the specific mechanisms of HXC on the SQBS treatment.

Since genomics, transcriptomics and proteomics could not meet the demand of interpreting the intricate responses of organisms following physiological and pathophysiological disturbances, as a novel "omics" and rapid-developing technology, metabolomics has emerged as a powerful means to explore complicated systems and assist with medical modernization. ${ }^{12}$ It can monitor and quantify the changes in small molecule metabolites, such as fatty acids, amino acids, peptides, nucleic acids, organic acids, vitamins, and carbohydrates, in an organism or a biological sample during the ultimate response to the endogenous and exogenous factors. ${ }^{13}$ Metabolomics can systematically and holistically probe the metabolome and metabolic pathway variations to explore the efficacy of a therapy, particularly that of TCM, which is difficult to study using conventional pharmacological methods due to its multi-component and multi-target peculiarity. ${ }^{\mathbf{1 4}}$ Currently, metabolomics has been successfully applied to detect metabolic changes in sepsis and characterize biochemical pathways. Metabolomics-guided biochemical analysis provides us with the further understanding of specific signaling pathways. Some studies reported that sepsis survivors exhibited a metabolic signature, characterized by the decreased level of glucose, maltose, tyrosine, beta-alanine, acetate, glutamine, and succinate. ${ }^{15-17}$ The combined approach of high-throughput techniques contribute to revealing the hidden features of sepsis. The main aim of our study is to preliminarily discuss the capability of the metabolomics approach and explore the molecular mechanism in order to treat SQBS in rats, with an attempt to bring new ideas and fundamental reference material for the future sepsis clinical treatment.

\section{Materials and methods}

\subsection{Experimental materials}

A biological safety cabinet was purchased from OLYMPUS, Japan. A low speed desktop centrifuge (TDZ5-WS) was purchased from Bruker, Germany. An electrothermal and thermostatic water tank was purchased from Thermo Company. A precision electronic balance was purchased from Qilinbeier Company (GL-802B $10 \mathrm{mg}$ to $1000 \mathrm{~g}$ ). Blood viscosity meter (LBY-N6), red blood cell function laser diffractometer (LBY-BX2) and platelet aggregation instrument (LBY-NJ2) were obtained from Beijing Pulisheng Electronics Group Co., Ltd. The image analysis software used in the study was Image-Pro Plus 5.0 with a digital camera (DXM1200; Nikon).

\subsection{Chemicals and reagents}

The prothrombin time (PT), activated partial thromboplastin time (APTT) and thrombin time (TT) assay kits were obtained from Wuhan Jieyangsheng Science and Technology Ltd. The alanine amino-transferase (ALT), aspartate aminotransferase (AST), total bilirubin (T-BIL) and total bile acid (TBA) assay kits were purchased from Sinopharm Chemical Reagent Co., Ltd. (Shanghai, China). The urea and carnitine ( $\mathrm{Cr}$ ) assay kits were purchased from Nanjing Jiancheng Bioengineering Institute (Nanjing, China). The C-reactive protein (CRP), lipopolysaccharide (LPS) and lipopolysaccharide-binding protein (LBP) assay kits were bought from Senbeijia Bioengineering Institute (Nanjing, China). The monocyte chemoattractant protein-1 (MCP-1) assay kit was purchased from Sigma (St. Louis, Mo, USA). HPLC grade acetonitrile, methanol and formic acid were purchased from Merck (Darmstadt, Germany). Distilled water (18.25 M $\Omega$ ) for the mobile phase and the aqueous solutions was purchased from Sabinsa Corporation (Piscataway, USA). Leucine enkephalin was purchased from Sea Sky Bio Technology Co. Ltd. (Bei-jing, China). Prednisone, pentobarbital sodium and physiological saline solution were purchased from Sigma Aldrich (Saint Quentin Fallavier, France). Huaxian capsule was purchased from Tong Ren Tang Chinese Medicine Co., Ltd. (Beijing, China). Other reagents and chemicals were of analytical grade throughout the experiment.

\subsection{Animal handling and model establishment}

Male Wistar rats (weighing $220 \pm 10 \mathrm{~g}$ ) free from specific pathogens were purchased from the animal center of the National Institute for the Control of Pharmaceutical and Biological Products, Beijing, China (Catalog No. 01016711). The rats were raised in an animal room with a $12 \mathrm{~h}$ light/dark cycle at a constant temperature and humidity. Standard food and water were autoclaved and distributed freely every day. Rats were divided into the control, model and HXC-treated groups with 8 animals in each group after acclimatizing for one week. Multiple-factor stimulation methods, including hunger, fatigue, cold and dampness, panic and high-fat diet, were adopted to establish the Qi deficiency and blood stasis syndrome model in one week. The performance of rats was observed. On the seventh day, the rats were injected with $4 \mathrm{mg}$ $\mathrm{kg}^{-1}$ LPS (isotonic $\mathrm{NaCl}$ solution diluted to $1 \mathrm{~mL}$ ) into the tail vein. After $24 \mathrm{~h}$ of injection, the rats in the HXC-treated group were administered HXC orally at a concentration of $0.8 \mathrm{~g} \mathrm{~kg}^{-1}$ once a day for two weeks. The rats in the control and model groups were given the same volume of normal saline. The experimental procedures were approved by the Animal Care and Ethics Committee at Heilongjiang University of Chinese Medicine, and all experiments were performed in accordance with the declaration of Helsinki.

\subsection{Sample collection and preparation}

After two consecutive weeks of drug administration as described above, $2 \mathrm{~mL}$ blood was taken from the eyelids, anticoagulated with $3.8 \%$ sodium citrate and then centrifuged at $2500 \mathrm{rpm}$ for $15 \mathrm{~min}$. The obtained plasma was evaluated for coagulation, including the PT, APTT, and TT tests. All the surviving rats were sacrificed by exsanguination from the abdominal aorta under $4 \%$ isoflurane anesthesia. Blood was immediately collected in heparinized tubes. A fraction of the blood sample was used to measure various indices including the whole blood viscosity $\left(\eta_{\mathrm{b}}\right)$, plasma viscosity $\left(\eta_{\mathrm{p}}\right)$, hematocrit (HCT), erythrocyte aggregation index (EAI), erythrocyte rigidity exponent (ERI) and reduction viscosity (RV) in hemarheology. Another fraction of the blood sample was centrifuged at 
$3500 \mathrm{rpm}$ for $15 \mathrm{~min}$ at $4{ }^{\circ} \mathrm{C}$. The serum supernatant was obtained and stored immediately at $-80^{\circ} \mathrm{C}$ for biochemical analysis, such as ALT, AST, T-BIL, CRP, and LBP, as well as MCP-1 detection and UPLC-MS metabolomics analysis. For the UPLC-MS analysis, the serum samples were thawed at $4{ }^{\circ} \mathrm{C}$. Then, $200 \mu \mathrm{L}$ of the serum sample was washed with $500 \mu \mathrm{L}$ of methanol. The supernatant was transferred to an auto-sampler vial after centrifugation at $15000 \mathrm{rpm}$ for $15 \mathrm{~min}$ at $4{ }^{\circ} \mathrm{C}$. Heart, liver, lung and kidney were quickly removed and rinsed with cold phosphate buffered saline. After the organs were immersed in $12 \%$ neutral-buffered formaldehyde for $24 \mathrm{~h}$, they were embedded in paraffin and equably sliced into $5 \mu \mathrm{m}$-thick sections. Then, the slices were stained with haematoxylin and eosin (H\&E) for the detection of histopathological changes in different groups.

\subsection{Metabolomics analysis}

2.5.1 UPLC-MS detection conditions. UPLC UltiMate 3000 (Dionex Corporation, Sunnyvale, CA, USA) coupled with MS (5500 Q-
TRAP, AB SCIEX, USA) was used for the metabolic analysis. The chromatographic separation of samples was conducted using a 2.1 $\times 100 \mathrm{~mm}$ C18 reverse-phase column with a $1.8 \mathrm{~m}$ particle size (Waters Corp., Milford, MA, USA). Mass Hunter Qualitative Analysis Software (version B.03.01, Agilent Technologies) and EZinfo 2.0 software (Waters Corporation, Manchester, UK) were applied to process the UPLC-MS data. For the chromatographic separation, the column was set at $40{ }^{\circ} \mathrm{C}$ and the flow rate was $0.5 \mathrm{~mL} \mathrm{~min}{ }^{-1}$. The serum sample was eluted with a linear gradient of $2-99 \%$ of solution A, where solution A was methanol with $0.15 \%$ formic acid and solution B was water with $0.15 \%$ formic acid. The gradient elution conditions were the following: $0-2 \mathrm{~min}, 2 \%$ solution A; 2-5 min, 2$45 \%$ solution A; 5-8 min wash with $45-80 \%$ solution A; 8-10 min wash with $80-99 \%$ solution $\mathrm{A}$; and a 5 min recycling time at the flow rate of $150 \mu \mathrm{L} \mathrm{min}{ }^{-1}$. The MS system was equipped with an ESI ion source that operated both in positive ionization $\left(\mathrm{ESI}^{+}\right)$and negative ionization modes $\left(\mathrm{ESI}^{-}\right)$. In the $\mathrm{ESI}^{+}$mode, the capillary voltage was $3800 \mathrm{~V}$, the sampling cone voltage was $50 \mathrm{~V}$, the source temperature

\section{Control group Model group HXC group}

Heart
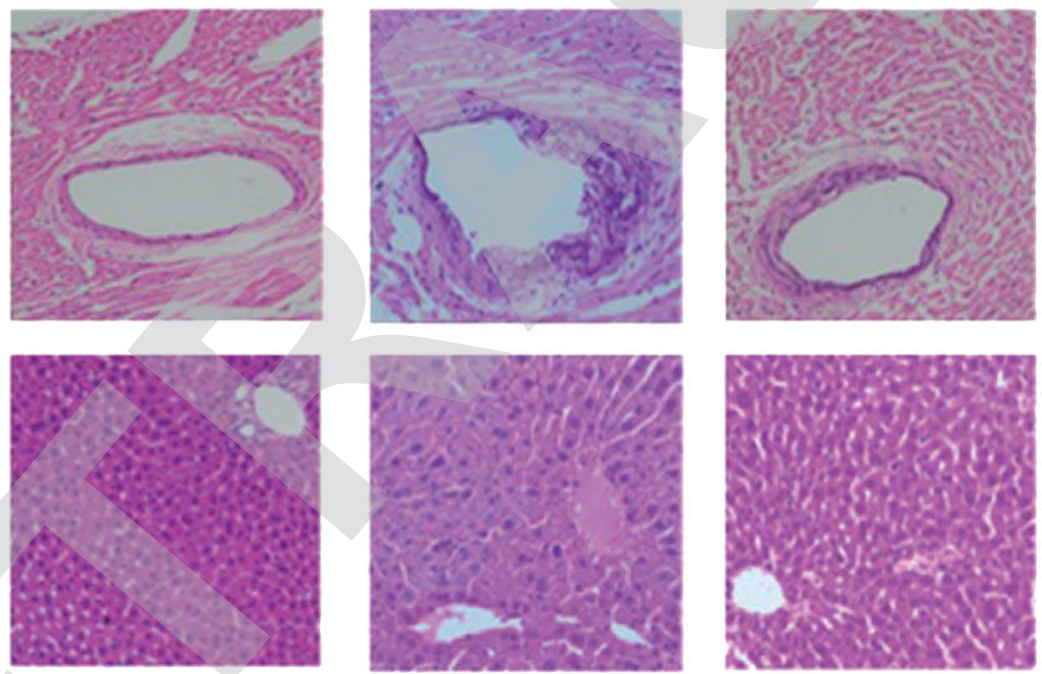

Liver
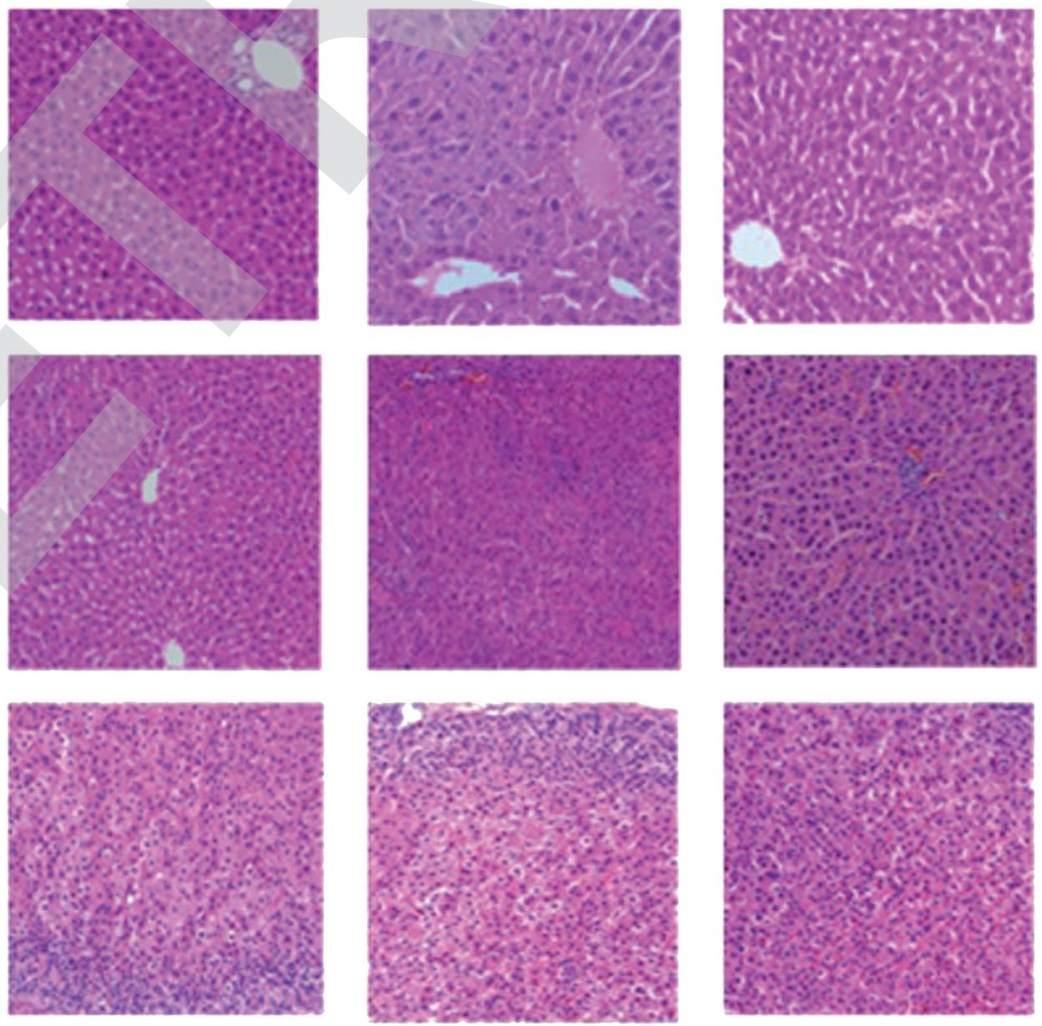

Fig. 1 Pathological changes in the heart, liver, lung and kidney of rats in different groups. 
was $130{ }^{\circ} \mathrm{C}$, the desolvation temperature was $400{ }^{\circ} \mathrm{C}$, the cone gas flow was $55 \mathrm{~L} \mathrm{~h}^{-1}$, and the desolvation gas flow was $500 \mathrm{~L} \mathrm{~h}^{-1}$. The $\mathrm{ESI}^{-}$mode conditions were the following: capillary voltage $2500 \mathrm{~V}$, sampling cone voltage $45 \mathrm{~V}$, source temperature $120^{\circ} \mathrm{C}$, desolvation temperature $330{ }^{\circ} \mathrm{C}$, cone gas flow $50 \mathrm{~L} \mathrm{~h}^{-1}$, and desolvation gas flow $450 \mathrm{~L} \mathrm{~h}^{-1}$. The data acquisition rate was set to $0.45 \mathrm{~s}$ with a $0.1 \mathrm{~s}$ inter scan delay. The lock-mass of leucine enkephalin at $50 \mathrm{fmol}$

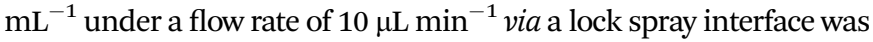
used to ensure the accuracy and reproducibility during all analyses, and all data were collected in a continuum mode from 50 to $1000 \mathrm{~m} /$ $z$ for the positive mode $[\mathrm{M}+\mathrm{H}]^{+}=556.2771$ and the negative mode $[\mathrm{M}-\mathrm{H}]^{-}=554.2615$; the frequency of the lock spray was maintained at $11 \mathrm{~s}$.

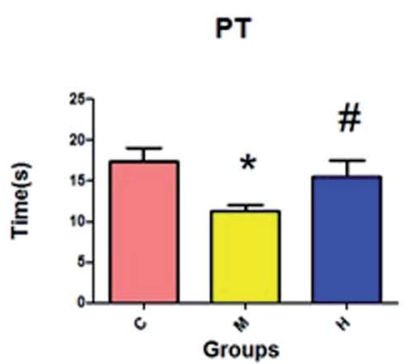

пр

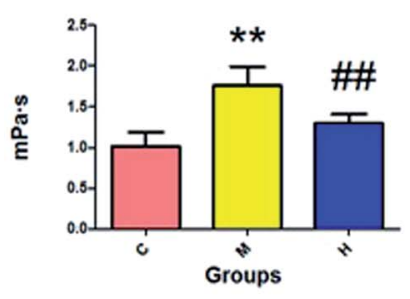

RV

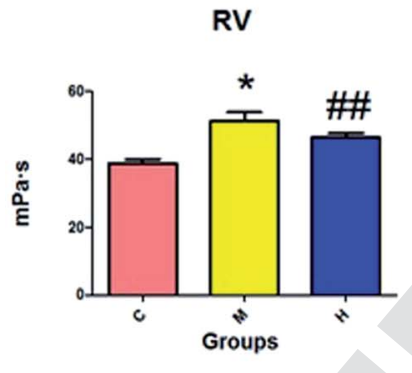

TBA

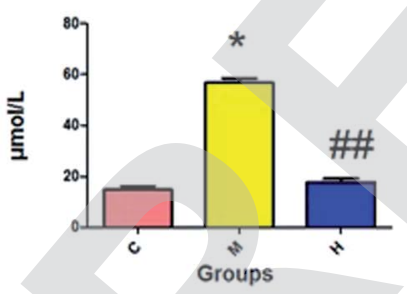

LPS

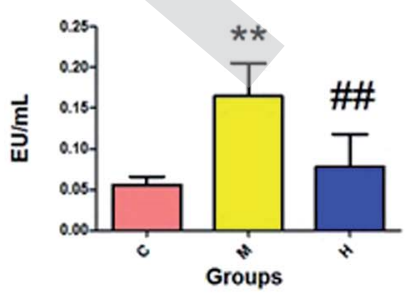

APTT

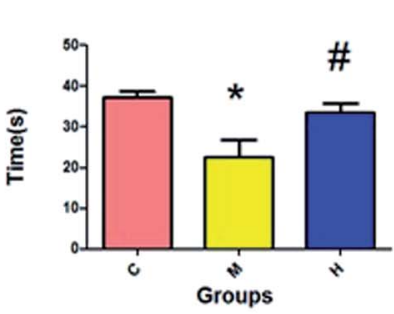

HCT

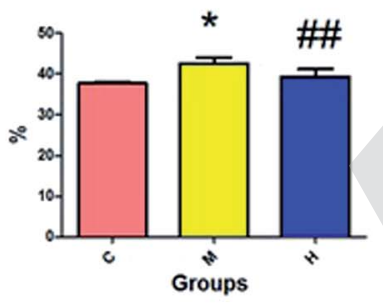

ALT

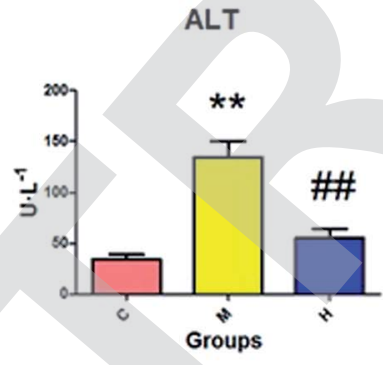

Urea

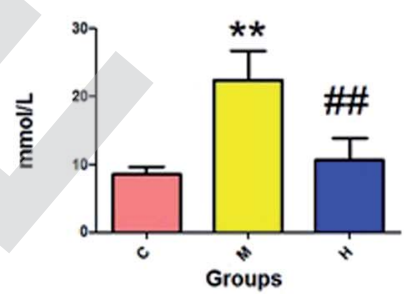

LBP

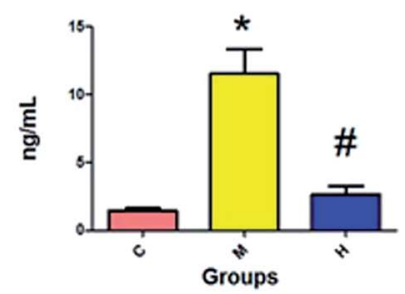

TT

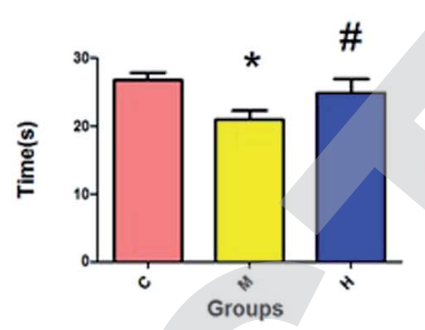

EAI

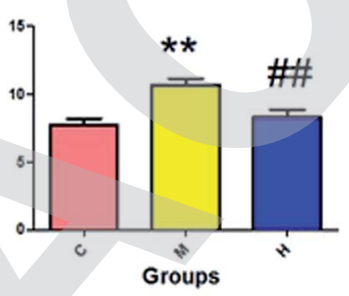

AST

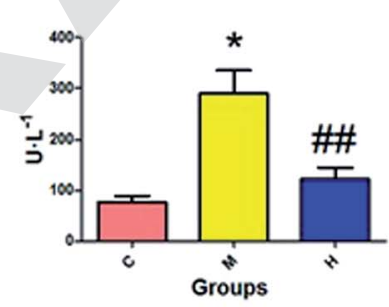

$\mathrm{Cr}$
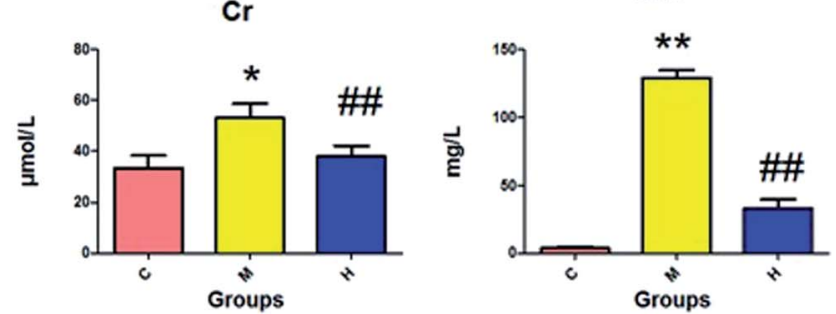

MCP-1

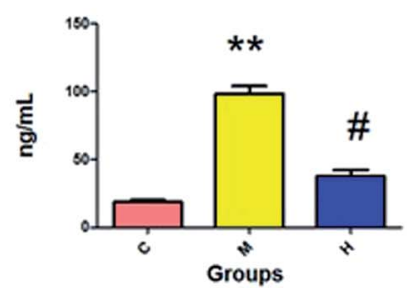

Fig. 2 Biochemical index detection of the SQBS. C: control group; M: model group; H: huaxian capsule group. 
2.5.2 Data processing and interpretation. Prior to performing the multivariate data analysis, all the raw MS data variables were processed by MarkerLynx Application Manager (Waters Corp.). The gained data matrix was produced with associated retention time (Rt) and accurate mass $(\mathrm{m} / \mathrm{z})$, and the normalized chromatographic peak area was calculated with a single accurate mass, and then imported into the EZinfo2.0 software for exploring the metabolome similarities and differences in different groups. As an exploratory unsupervised pattern recognition method, principal component analysis (PCA) has the ability to transform the variables into a smaller number of new latent variables, which congregate when metabolomic ingredients exhibit higher similarity and vice versa. Orthogonal partial least-squares discriminant analysis (OPLS-DA) was applied to reduce the risk of the counterfeit separation of the categories and highlight the discrepancy among the groups after further validating the supervised model by the permutation test of $R_{2}, Q_{2}$ and the misclassification rate calculation. After the OPLS analysis, the S-plots and VIP plot were generated in accordance with their contribution to the change and correlation ratio of the disorder in metabolic profiling. $P$-Value presents a positive correlation to the contribution ratio between the control group and the model group. A metabolite with a VIP value greater than 1 and a $P$-value smaller than 0.05 in Student's $t$ test was deemed as the potential metabolite closely associated with SQBS; its chemical structure and biological parameters were determined using basic information about Rt, precise molecular
(A)

\section{Positive mode}

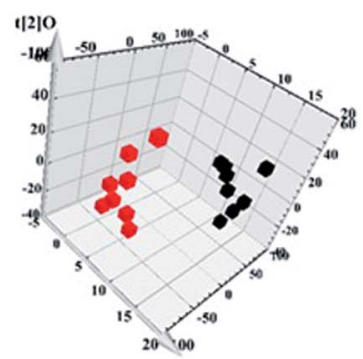

(B)

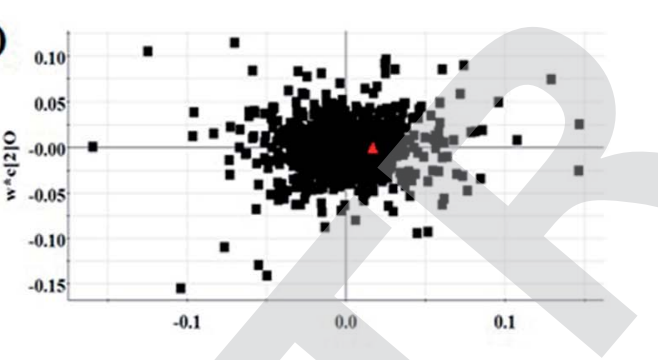

(C)

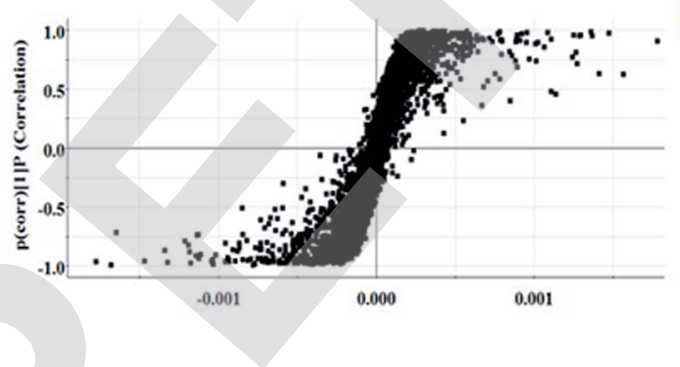

(D)

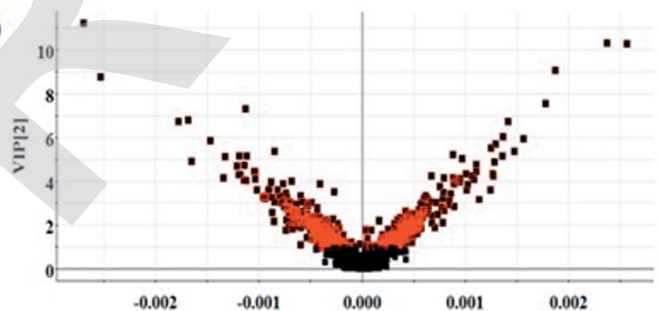

$\left(\mathbf{A}^{\prime}\right)$

\section{Negative mode}

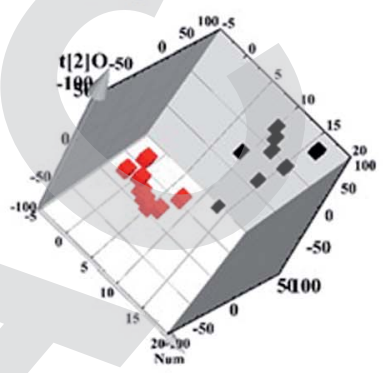

(B')

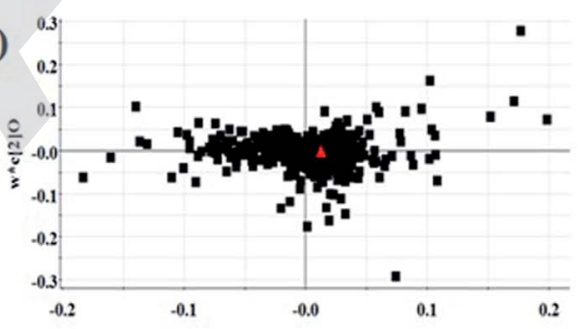

$\left(C^{\prime}\right)$

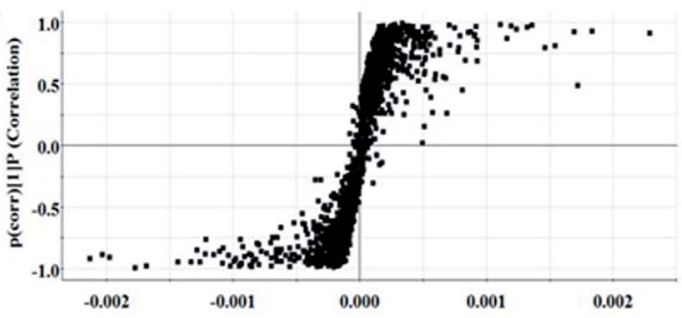

(D')

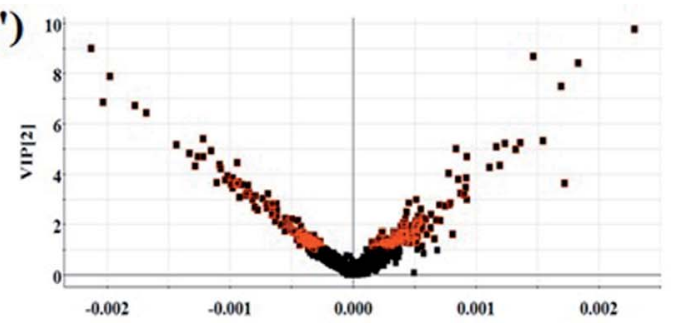

Fig. 3 Multivariate analysis of metabolites in the SQBS rat. (A) and ( $\left.A^{\prime}\right)$ 3D OPLS-DA score plot of the serum metabolite clustering for the control and model groups in both ion modes. (B) and ( $\left.\mathrm{B}^{\prime}\right)$ Loading plot of the OPLS-DA model serum metabolite clustering for the control and model groups in both ion modes. (C) and ( $\left.C^{\prime}\right)$ S-plot of the OPLS-DA model serum metabolite clustering for the control and model groups in both ion modes. (D) and ( $\left.D^{\prime}\right)$ VIP-plot of the OPLS-DA model serum metabolite clustering for the control and model groups in both ion modes. 
mass coupled with the MS/MS fragments data and the online databases, such as ChemSpider (http:/www.chemspider.com/), HMDB (http://www.hmdb.org/), KEGG (http://www.genome.jp/ kegg/), METLIN (http://metlin.scripps.edu/), and Lipid Maps (http://dev.lipidmaps.org). The relative signal intensities of selected serum metabolites of different groups were calculated to reveal the metabolic regulation effect of HXC on the SQBS rats. The metabolite biomarkers under the control of HXC were imported into the MetaboAnalyst 4.0 (http://www.metabo analyst.ca/) and the ingenuity pathway analysis (IPA) software to gain insight into the concentration change, interaction, metabolic pathway and network.

\section{Results}

\subsection{Conventional observation of animal model}

After modeling, the rats in the model group gradually exhibited sluggish eyes, shortness of breath, astasia, lacklustre pelage, light-colored claws, purple and dark tongue, tail with congestion points from the tip to the root, inactivity, slow response to the outside world, viscous secretions coming from the eyelids, fecal adhesions in the anus and cloudy urine. The anatomy analysis showed that the abdominal cavity was filled with a turbid exudate and intestinal adhesions. After the administration of HXC on the 8 th day, the above symptoms gradually improved. In the control group, the rats were fairly active with lustrous pelage and the tails without congestion. The body mass gradually increased and no other abnormalities were observed.

\subsection{Pathological observations}

Histology results showed a normal cell structure in the liver, lung and kidney tissues of rats in the control group. The endothelial cell membrane of the heart tissue was intact, and the myocardial cells and fiber bundles were arranged neatly. Hepatic sections of the model group rats showed increased inflammatory cell infiltration, accompanied by moderate portal inflammation and hepatocellular necrosis. Lung and kidney tissues presented histopathological changes, such as edema, inflammatory cell infiltration and interstitial inflammation. Endothelial cells of the heart in the model group appeared irregularly arranged, exhibited shedding, a small amount of lipid deposited and a certain degree of myocardial fibrosis. These pathological changes in different organs were significantly relieved by $\mathrm{HXC}$, as shown in Fig. 1.

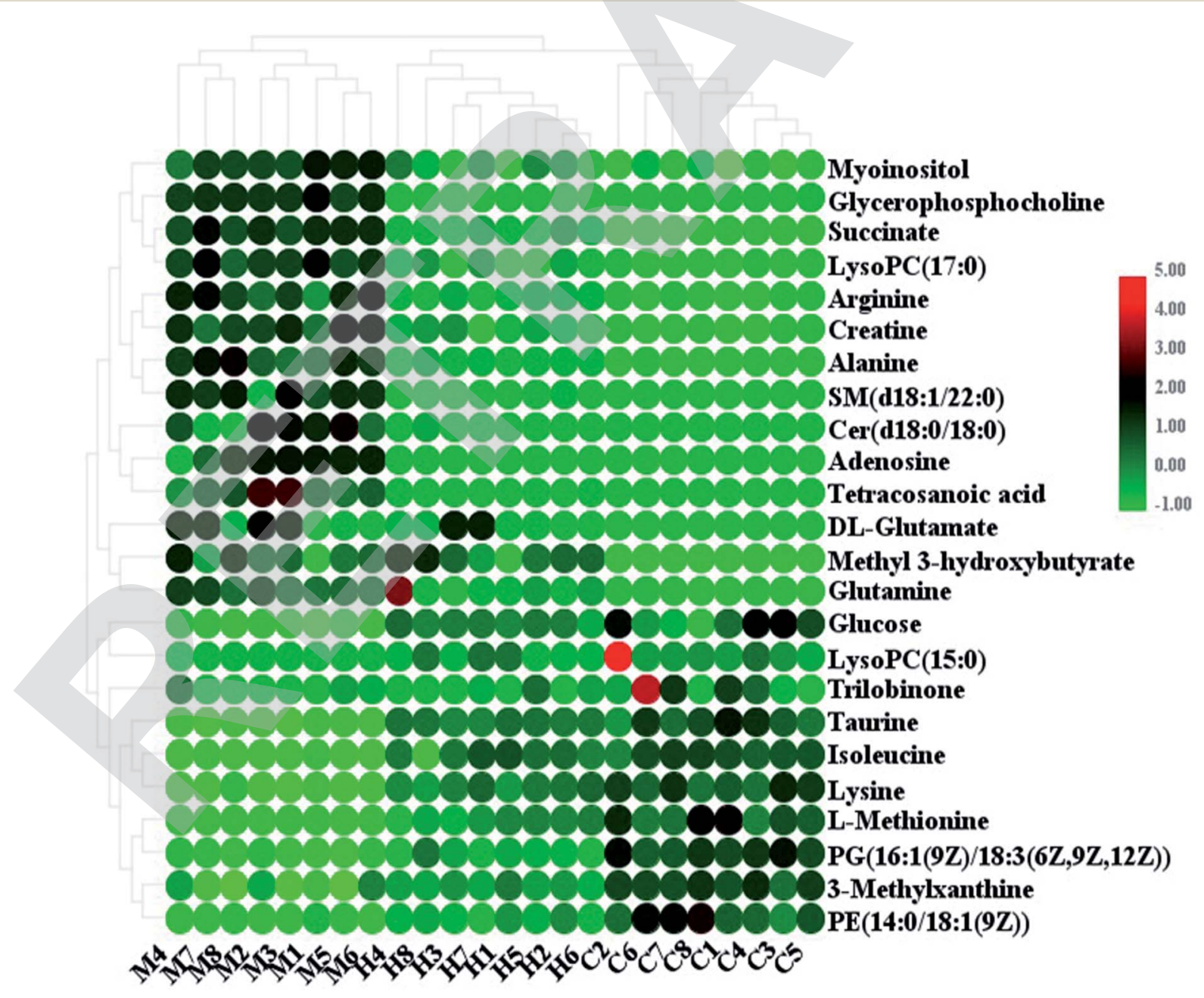

Fig. 4 Heatmap of significant changes in potential biomarker candidates between control, model and HXC groups. 


\subsection{Biochemical index analysis}

The amount of PT, APTT, and TT in the serum of the model group was significantly decreased $(P<0.05)$ compared to that in the control group, while there was an obvious increase in the other biochemical variables $(P<0.05$ or $P<0.01)$, as shown in Fig. 2 . The distinct changes between the control and model groups indicated that biological turbulences may have occurred in the SQBS rats. The level of PT, APTT, and TT in the HXC group markedly increased $(P<0.05)$ compared to that in the model group, the amount of ERI, LBP and MCP-1 evidently increased $(P$ $<0.05)$, and other biochemical indices notably increased $(P<$ 0.01). These data confirmed that the HXC treatment contributed to an outstanding improvement in the SQBS rat condition.

\subsection{Multivariate analysis of the UPLC-MS data}

According to the UPLC-MS conditions described above, all the data from representative UPLC-MS spectra of the serum samples were imported into the Progenesis QI software for multiple statistical analyses. The trajectory of 2D PCA scores for the control, model and HXC groups in both the positive (Fig. S1A $\dagger$ ) and negative modes (Fig. S1B $\dagger$ ) were clearly separated, with the model group trajectory being closer to that of the HXC group. Furthermore, the metabolome differences between the control and model groups could be distinguished in the 3D OPLS-DA score plot (Fig. 3A and $\mathrm{A}^{\prime}$ ), where the separation indicated that the serum sample of rats after the model establishment exhibited metabolome changes. In the corresponding loading-plots (Fig. 3B and $\mathrm{B}^{\prime}$ ), the ions of metabolites furthest away from the origin are likely to be considered as the differentiating metabolites. The S-plot (Fig. 3C and $\mathrm{C}^{\prime}$ ) and the VIP score plot (Fig. 3D and $\mathrm{D}^{\prime}$ ) obtained after the OPLS-DA analysis indicated that the distance of ions from the origin have a positive correlation with the alteration of metabolic trajectory, and the contribution of the cluster and discrimination variables was applied for the visualization and discovery of different metabolites.

\subsection{Biomarker identification}

The significant variables in each of the multivariate analysis models combined with the high-resolution MS data were selected for the unknown endogenous biomarker prediction. First, the $P$ values $<0.05$ and VIP $>1$ were considered to be objective indicators of the metabolite expression. Then, the information about the predicted potential biomarkers in line with the exact $m / z$ from significant peaks was provided by online database retrieval. The RT $-m / z$ pairs of selected differential ions from each of the two groups were matched and identified using the tandem MS fragmentation with the reference standards and the database. When
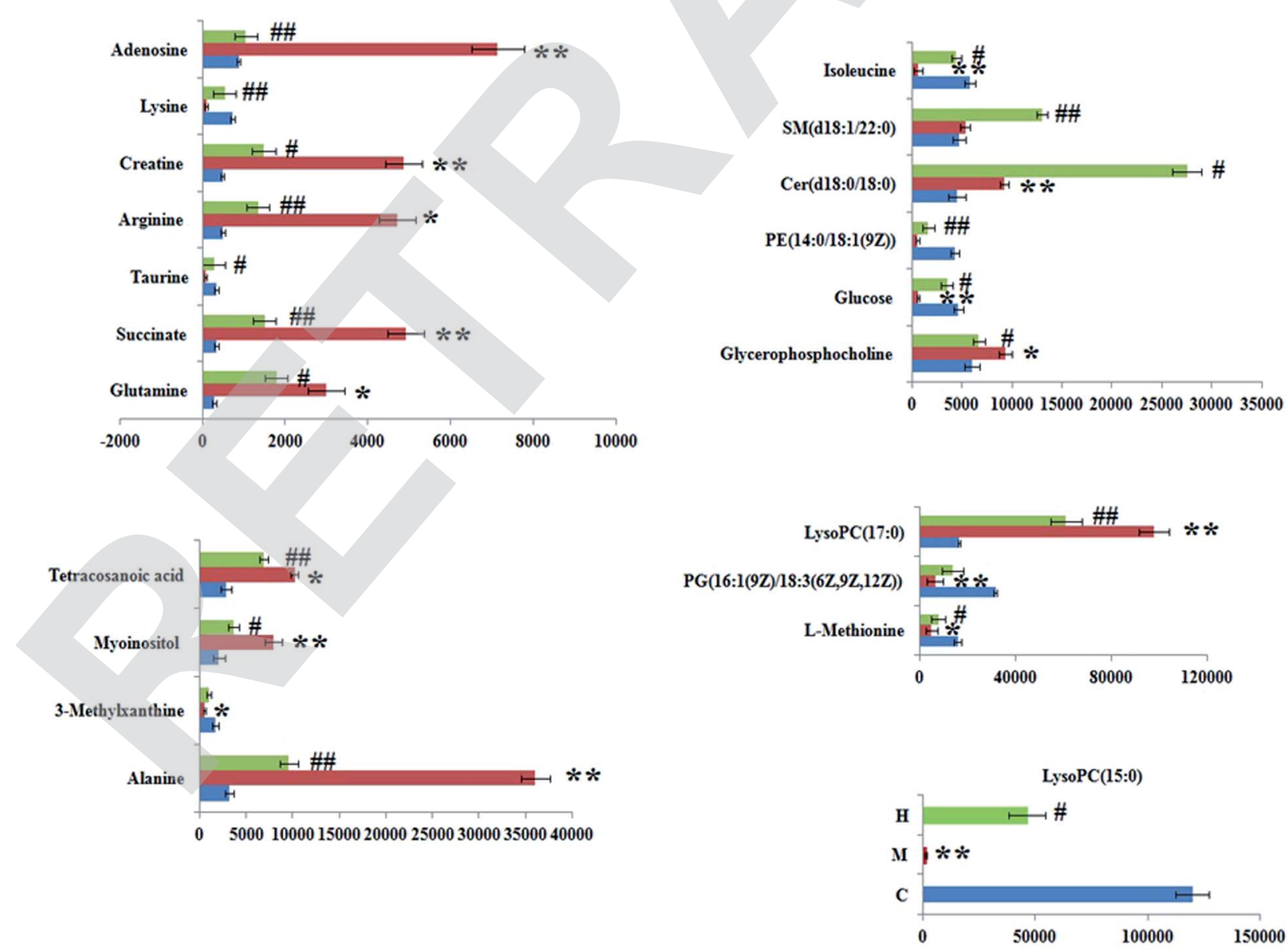

Fig. 5 Relative UPLC-MS signal intensity of serum metabolites in different groups. 
a mass difference between the observed and the theoretical mass was $<10 \mathrm{ppm}$, the metabolite name was reported. A total of 24 potential metabolites were identified related to the morbidity of the SQBS between the control and model groups (Table S1 $\dagger$ ), in which 21 of them were isoleucine, alanine, lysine, L-methionine, glutamine, 3-methylxanthine, adenosine, myoinositol, succinate, PG(16 : 1(9Z)/18 : 3(6Z,9Z,12Z)), glycerophosphocholine, taurine, arginine, creatine, glucose, LysoPC(17:0), PE(14:0/18:1(9Z)), Cer(d18:0/18:0), SM(d18:1/22:0), LysoPC(15:0), and tetracosanoic acid; they were remarkably regulated by the HXC treatment. As shown in Fig. 4, the color brightness difference presents the concentration change of the biomarker. The relative signal intensities of serum metabolites in different groups identified by UPLC-MS reflect the extent of the regulating action of HXC (Fig. 5).

\subsection{Metabolic pathway and network construction}

MetPA, a free web-based tool with a pathway topology analysis based on the MetaboAnalyst platform, is shown in Fig. 6A. The selected metabolite biomarkers, managed by the HXC treatment, were mainly involved in the following pathways: taurine and hypotaurine metabolism, D-glutamine and D-glutamate metabolism, alanine, aspartate and glutamate metabolism, lysine degradation, glycerophospholipid metabolism, starch and sucrose metabolism, inositol phosphate metabolism, lysine biosynthesis, amino sugar and nucleotide sugar metabolism, and aminoacyl-tRNA biosynthesis. After the HXC administration, the KEGG global metabolic network that portrays metabolites and enzyme/KEGG orthologs, associated with 10 metabolites in 9 pathways was plotted (Fig. 6B). Metabolite-metabolite interaction network was involved in the glutamine, taurine, L-methionine, creatine, lysine and isoleucine potential functional relationship (Fig. S2†), which is in line with the data for the reactions, involving similar chemical structures and molecular activities. IPA that generated 13 networks has revealed amino acid metabolism, molecular transport, small molecular biochemistry, cell signaling as well as vitamin and mineral metabolism fluctuation after the HXC administration (Fig. 7).

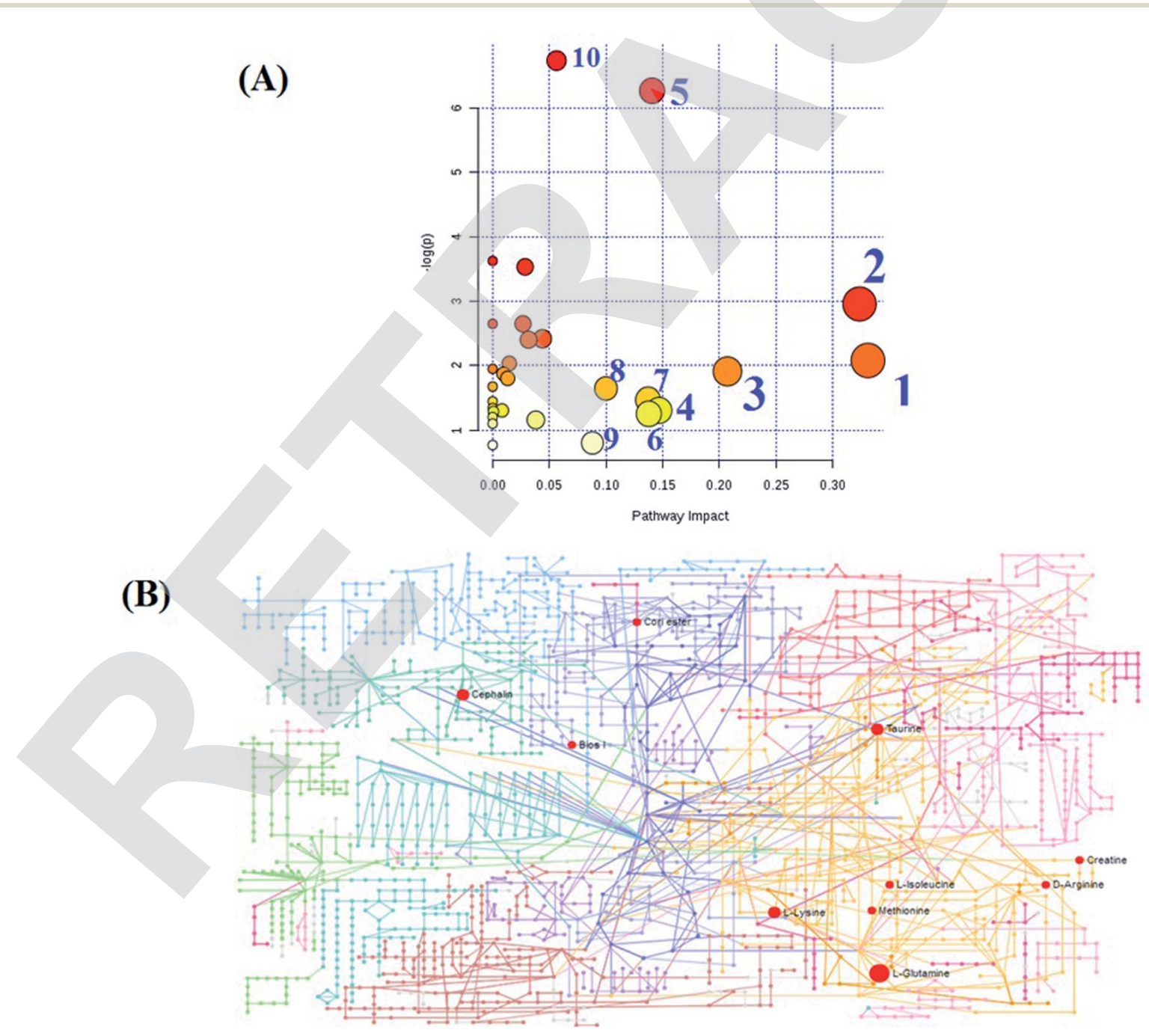

Fig. 6 Altered metabolomic pathways observed in the serum sample data after the huaxian capsule treatment (A). KEGG global metabolic network associated with the huaxian capsule protective activity towards the SQBS rats (B). 


\section{Discussion}

Experimental animal science is an important part of modern science and the foundation of life science. With further detailed research on the basic theory of TCM, the development of TCM animal models is becoming increasingly vital and urgent. ${ }^{18}$ It is difficult to carry out theoretical TCM studies using traditional animal models of modern diseases, and the existing research of animal model has been stuck in the arduous stage for long time, inhibiting the development of the in-depth practical applications. The key to solving this problem is to study the combination of the disease and syndrome animal models, making the simultaneous application of multiple model replication methods inevitable. According to TCM, Qi deficiency and blood stasis play a critical role in sepsis, which is caused by blood and vitality losses, cold invasion and depression. This experiment attempts to simulate the pathogenetic process of a disease, which first induces extreme fatigue in the body and spirit of an animal, Qi deficiency and blood stasis. Then, stimulates acute systemic inflammatory response to sepsis via LPS injection. Compared with other methods of sepsis modeling, LPS injection can make animals exhibit high-level and lowresistance hemodynamic changes and high metabolic status for a long time, which is similar to the pathophysiology and clinical manifestation of sepsis in patients.

The above results show that the general observation of the rat represents the main features of Qi deficiency after modeling. The coagulation function indices, such as PT, APTT and TT, decreased compared with those of the control group, and the hemarheology indices, including $\eta_{\mathrm{b}}, \eta_{\mathrm{p}}$, HCT, EAI, ERI and RV, significantly increased, indicating that the exogenous and endogenous coagulation system disorders had occurred in rats. $^{19,20}$ HCX effectively increased the coagulation function indices and reduced the hemorheological parameters, thus

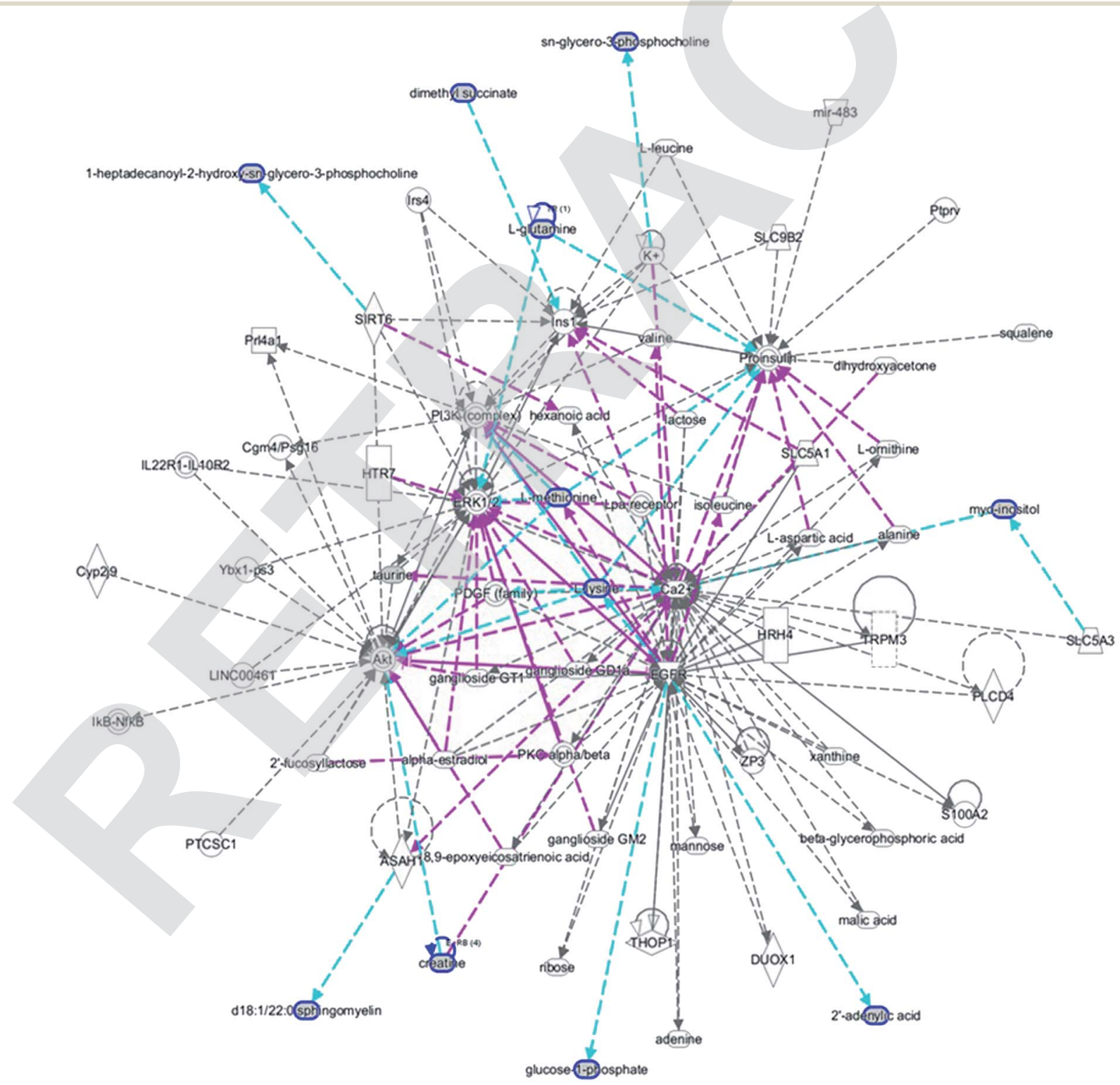

Fig. 7 IPA prediction networks related with huaxian capsule protective activity on the sepsis of Qi deficiency and blood stasis syndrome rat in this study. 
exhibiting an anticoagulant effect and improving the state of blood concentration, adhesion, coagulation and aggregation. ALTS, AST, T-BIL, TBA, urea, and Cr are common clinical indicators of the important organ dysfunction, such as liver, lung and kidneys, and the up-regulated level of LPS, LBP, CRP, and MCP-1 is closely associated with sepsis. ${ }^{21}$ After the CHM intervention, all of the increased indicators were significantly down-regulated, indicating that HXC had a significant therapeutic effect on the SQBS rat condition. The metabolic profile changes indicate that HXC interferes at the molecular level. Based on the profiles of twenty-four differential metabolites, differences existed between the blank and model groups, and HXC had a regulatory effect on 21 of them. The therapeutic activity of HXC primarily affected the amino acid metabolism. Taurine is a sulfur-containing amino acid similar to methionine, cystine, cysteine and homocysteine. Because it is not a common structural building block of proteins, it is a lesserknown amino acid. The low levels of taurine in plasma have been found in a variety of conditions, such as depression, hypertension, hypothyroidism, gout, infertility, obesity, and kidney failure. The level of taurine in the model group increased after the HXC treatment, indicating that the HXC treatment had a regulatory effect on taurine metabolism. ${ }^{22}$ It was reported that glutamine protects against the myocardial damage, liver and kidney damage, and intestinal disorders in sepsis. Arginine and glutamate are the synthetic components of glutamine in the body and clinical immunomodulators in humans. In the model group, the levels of arginine and glutamate were abnormally higher than normal, indicating that the viscera of the model rats were damaged, which is consistent with the pathological observations. HXC can improve the abnormal metabolism of amino acids, such as glutamine, glutamic acid, alanine and aspartic acid. In addition, HXC regulates the metabolism of phospholipids, such as glycerophospholipids and inositol phospholipids, and protects against SQBS by regulating the serum PE(14:0/18:1(9Z)), LysoPC(15:0), glycerophosphocholine and inositol content. ${ }^{23}$

The rapidly growing field of metabolomics has shown significant potential to utilize the complicated TCM syndrome as a diagnostic tool and change the clinical practice of Chinese compound formulae. ${ }^{24-28}$ In contrast to other approaches, such as genomics, transcriptomics, and proteomics, metabolomics comes closest to the phenome accounting for the direct relationship between an individual, environment, and a pathogen. Precisely these composite metabolic characteristics lead to its utility in the evaluation of sepsis subtypes and contribute to the TCM development process. As the field evolves, focus will shift to better stratification of patients with sepsis subtypes, drug response prediction and effective constituent by metabolomics. ${ }^{29}$ The integration of these approaches could bring out a substantial challenge but also a huge probability for advances in solving the problems in the TCM field. ${ }^{30-34}$ Currently, the work process of metabolomics is tedious, which is adverse to real-time data collection used for therapeutic schedule making in clinical practice. The qualitative and quantitative identification of metabolites and pathways will be indispensable to the TCM drug response and syndrome analysis.

\section{Conclusion}

A serum metabolomics study was performed to characterize the changes in the metabolic regulatory biomarkers and pathways in the SQBS rats following the HXC intervention. The identified metabolite biomarkers indicated that multiple metabolic pathways were altered in the SQBS rats, as the disrupted amino acid, phospholipid, and energy metabolism was observed. The metabolomics study coupled with other parameters verified the therapeutic efficacy of HXC in ameliorating SQBS, which further deepens our understanding of SQBS and the pharmacodynamic mechanism of HXC. It also confirms the feasibility of using the metabolomics approach to decipher the therapeutic effects and intricate mechanisms of TCM on the treatment of complicated diseases.

\section{Conflicts of interest}

The authors declare no competing interests.

\section{Acknowledgements}

This work was supported by grants from the Key Program of Natural Science Foundation of State (Grant No. 81774276, 81470196).

\section{References}

1 L. Wang, H. Zhao and D. Wang, Inflammatory cytokine expression in patients with sepsis at an intensive care unit, Exp. Ther. Med., 2018, 16(3), 2126-2131.

2 H. Brodska, J. Valenta, K. Pelinkova, et al. Diagnostic and prognostic value of presepsin vs. established biomarkers in critically ill patients with sepsis or systemic inflammatory response syndrome, Clin. Chem. Lab. Med., 2018, 56(4), 658-668.

3 J. Duran-Bedolla, M. A. Montes de Oca-Sandoval, V. SaldañaNavor, et al. Sepsis, mitochondrial failure and multiple organ dysfunction, Clin. Invest. Med., 2014, 37(2), E58-E69.

4 M. Hayakawa, K. Yamakawa, D. Kudo, et al. Optimal Antithrombin Activity Threshold for Initiating Antithrombin Supplementation in Patients With SepsisInduced Disseminated Intravascular Coagulation: A Multicenter Retrospective Observational Study, Clin. Appl. Thromb./Hemostasis, 2018, 24(6), 874-883.

5 S. Lepine, B. Lawton, S. Geller, et al. Severe maternal morbidity due to sepsis: The burden and preventability of disease in New Zealand, Aust. N. Z. J. Obstet. Gynaecol., 2018, 58(6), 648-653.

6 Q. Deng, X. Zhou and F. Wu, Updated Knowledge About the Diagnosis and Treatment of Sepsis and Septic Shock, J. Emerg. Nurs., 2018, 44(5), 444-445.

$7 \mathrm{~W}$. Liu and Y. Liu, Youyou Tu: significance of winning the 2015 Nobel Prize in Physiology or Medicine, Cardiovasc. Diagn. Ther., 2016, 6(1), 1-2.

8 M. Zhang, C. S. Peng and X. B. Li, In vivo and in vitro metabolites from the main diester and monoester diterpenoid alkaloids in a traditional Chinese herb, the 
aconitum species, J. Evidence-Based Complementary Altern. Med., 2015, 2015, 252434.

9 S. T. Ma, H. Yu, X. L. Zhang, et al. Potency Material Bases of Xuebijing Formula and Its Multi-target Effects on Sepsis, Zhongguo Zhongxiyi Jiehe Zazhi, 2015, 35(11), 1351-1355.

10 X. Liang, M. Zhou, X. Y. Ge, et al. Efficacy of traditional Chinese medicine on sepsis: a systematic review and MetaAnalysis, Int. J. Clin. Exp. Med., 2015, 8(11), 20024-20034.

$11 \mathrm{~J}$. Cao, Z. Chen, Y. Zhu, et al. Huangqi-Honghua combination and its main components ameliorate cerebral infarction with Qi deficiency and blood stasis syndrome by antioxidant action in rats, J. Ethnopharmacol., 2014, 155(2), 1053-1060.

12 Y. F. Li, S. Qiu, L. J. Gao, et al. Metabolomic estimation of the diagnosis of hepatocellular carcinoma based on ultrahigh performance liquid chromatography coupled with time-offlight mass spectrometry, RSC Adv., 2018, 8(17), 9375-9382.

$13 \mathrm{H}$. Du, X. Zhao and A. Zhang, Identifying potential therapeutic targets of a natural product Jujuboside B for insomnia through network pharmacology, Plant Sci. Today, 2014, 1(2), 69-79.

14 Y. Zhang, P. Liu, Y. Li and A. H. Zhang, Exploration of metabolite signatures using high-throughput mass spectrometry coupled with multivariate data analysis, $R S C$ Adv., 2017, 7(11), 6780-6787.

15 V. Bakalov, R. Amathieu, M. N. Triba, et al. Metabolomics with Nuclear Magnetic Resonance Spectroscopy in a Drosophila melanogaster Model of Surviving Sepsis, Metabolites, 2016, 6(4), E47.

16 X. Wang, J. Li and A. H. Zhang, Urine metabolic phenotypes analysis of extrahepatic cholangiocarcinoma disease using ultra-high performance liquid chromatography-mass spectrometry, RSC Adv., 2016, 6(67), 63049-63057.

17 V. Fanos, P. Caboni, G. Corsello, et al. Urinary (1)H-NMR and GC-MS metabolomics predicts early and late onset neonatal sepsis, Early Hum. Dev., 2014, 90(Suppl. 1), S78-S83.

18 S. Huang, F. Xu, Y. Y. Wang, et al. Improvement and application of an acute blood stasis rat model aligned with the 3Rs (reduction, refinement and replacement) of humane animal experimentation, Chin. J. Integr. Med., 2014, DOI: 10.1007/s11655-014-2008-y.

19 F. Ibrahim, L. Calmette, A. Layka, et al. PT, aPTT, TT and the hemostatic safety threshold of dabigatran and rivaroxaban, Ann. Biol. Clin., 2016, 74(4), 457-464.

20 D. Cao, C. Xu, Y. Xue, et al. The therapeutic effect of Ilex pubescens extract on blood stasis model rats according to serum metabolomics, J. Ethnopharmacol., 2018, 227, 18-28.

21 I. Herzum and H. Renz, Inflammatory markers in SIRS, sepsis and septic shock, Curr. Med. Chem., 2008, 15(6), 581-587.
22 W. Zhao, L. Jia, H. J. Yang, et al. Taurine enhances the protective effect of Dexmedetomidine on sepsis-induced acute lung injury via balancing the immunological system, Biomed. Pharmacother., 2018, 103, 1362-1368.

23 S. Cao, J. Ren, L. Sun, et al. Fish oil-supplemented parenteral nutrition prolongs survival while beneficially altering phospholipids' Fatty Acid composition and modulating immune function in rat sepsis, Shock, 2011, 36(2), 184-190.

24 A. Balasopoulou, G. P. Patrinos and T. Katsila, Pharmacometabolomics Informs Viromics toward Precision Medicine, Front. Pharmacol., 2016, 7, 411.

25 C. Lu, X. Zhao, Y. Li, et al. Serum metabolomics study of Traditional Chinese medicine formula intervention to polycystic ovary syndrome, J. Pharm. Biomed. Anal., 2016, 120, 127-133.

26 Q. Liang, Y. Zhu, H. Liu, B. Li and A. H. Zhang, Highthroughput lipidomics enables discovery of the mode of action of huaxian capsule impacting the metabolism of sepsis, RSC Adv., 2017, 7(71), 44990-44996.

27 Q. Liang, H. Liu, L. Xie, X. Li and H. Ai, High-throughput and multi-dimensional omics approach uncovers a huaxian capsule to ameliorate the dysregulated expression profiling of severe sepsis rats, RSC Adv., 2017, 7(32), 19894-19903.

28 Q. Liang, H. Liu, H. Xing, Y. Jiang and A. Zhang, UPLCQTOF/MS based metabolomics reveals metabolic alterations associated with severe sepsis, $R S C A d v$., 2016, 6, 43293-43298.

29 Q. Liang, H. Liu, X. Li and A. Zhang, High-throughput metabolomics analysis discovers salivary biomarkers for predicting mild cognitive impairment and Alzheimer's disease, $R S C A d v$. , 2016, 6, 75499-75504.

30 Y. Li, S. Qiu and A. Zhang, High-throughput metabolomics to identify metabolites serve as diagnostic biomarkers of prostate cancer, Anal. Methods, 2016, 8, 3284-3290.

31 Q. Liang, H. Liu, H. Xing, Y. Jiang and A. Zhang, Urinary UPLC-MS metabolomics dissecting the underlying mechanisms of huaxian capsule protects against sepsis, RSC Adv., 2016, 6, 40436-40441.

32 Q. Liang, H. Liu, H. Xing, Y. Jiang, T. Zhang and A. Zhang, High-resolution mass spectrometry for exploring metabolic signatures of sepsis-induced acute kidney injury, $R S C A d v$., 2016, 6, 29863-29868.

33 Q. Liang, H. Liu, Y. Jiang, H. Xing, T. Zhang and A. Zhang, Discovering lipid phenotypic changes of sepsis-induced lung injury using high-throughput lipidomic analysis, $R S C$ Adv. , 2016, 6, 38233-38237.

34 Q. Liang, H. Liu, T. Zhang, Y. Jiang, H. Xing and A. Zhang, Discovery of serum metabolites for diagnosis of mild cognitive impairment to Alzheimer's disease progression using an optimized metabolomics method, RSC Adv., 2016, 6, 3586-3591. 